\title{
RESEARCH
}

\section{Student Experiences With An Intervention Utilizing the Salience Principle to Reduce Psychological Attraction to Smartphones}

\author{
Elijah Myers, PharmD, Erin T. Drees, PharmD, Jeff Cain, EdD, MS \\ University of Kentucky, College of Pharmacy, Lexington, Kentucky
}

Corresponding Author: Jeff Cain, University of Kentucky, College of Pharmacy, 114M Lee T. Todd, Jr. Building, 789 South Limestone Dr., Lexington, KY 40536-0596. Tel: 859-257-4429. Email: jeff.cain@uky.edu

Submitted May 6, 2021; accepted August 27, 2021; ePublished September 2021

Objective. To determine pharmacy student reactions to and experiences with an intervention based upon the principle of salience to reduce psychological attraction and attention to smartphones.

Method. For a period of 3 weeks, participants were directed to change their smartphone's color setting to grayscale mode, turn off social media notifications, remove social media icons from smartphone home screen, and place the device away from bed when sleeping. A thematic analysis was performed on responses to an anonymous, open-ended survey question that asked participants to record any positive/negative changes to their life and well-being as a result of participating in the study.

Results. Thematic analysis revealed twenty unique themes, which provided a variety of notable observations including a reduction in both smartphone and social media use, reduced allure because of grayscale mode, frustrations caused by grayscale mode, increased productivity, and general improvements in sleep, face-to-face interactions, and overall wellbeing Conclusion. Results of this study provide initial evidence that changes to smartphone settings and physical placement at night may lead to positive outcomes. Participants reported primarily positive effects regarding well-being and reduced smartphone usage as a result of participating in the study. In many cases, less time on smartphones meant improved sleep, more productivity, more time for social/leisure activities, improved face-to-face interactions, and/or improvement in wellbeing.

Keywords: smartphones, social media, well-being, mental health, sleep

\section{INTRODUCTION}

Pharmacy educators have expressed concern over student well-being and advocated for intentional efforts to improve it. ${ }^{1-3}$ One such call has been to examine the potential negative effects that smartphones have on well-being ${ }^{4}$. A rise in reports of college student mental health issues that coincided with the rise in smartphone adoption has prompted some researchers to suggest a causative link. ${ }^{5}$ A specific area of concern is problematic smartphone use, defined as "an inability to regulate one's use of the mobile phone, which eventually involves negative consequences in daily life." ${ }^{26}$ The direction of causality is unknown, but research has shown correlation of problematic smartphone use with anxiety, depression, stress, and decreased well-being. ${ }^{7-10}$ Studies have also shown that preventative factors such as sleep are often negatively correlated with problematic smartphone use. ${ }^{11}$ Still, the associations between smartphone use and overall wellbeing are very nuanced. Why, how, and how much one uses smartphones may influence associated mental health outcomes. ${ }^{12-14}$ Certain specific uses of smartphones may even have a positive effect on well-being. ${ }^{15,16}$

Many students have realized an unhealthy attachment to their phones and expressed a desire to use them less often. ${ }^{17,18}$ Despite self-professed desire to decrease use, individuals may struggle to break or reduce the attachment. ${ }^{19}$ This may in part be due to the inherent design of these technologies, which use psychological processes to capture attention and promote frequent and/or sustained use. ${ }^{20}$ Many technology industry insiders have decried smartphone and social media companies use of psychological manipulation to draw and capture user attention and are now heralding approaches to curb "digital addictions". ${ }^{21}$ Interventions designed to help users control smartphone use have included both nontechnical (eg, digital detoxes) and technical (eg, mobile apps) measures, but there is a need for further exploration. ${ }^{22}$

Different types of studies examining numerous variables and conditions are needed to better understand the complex relationships between smartphones and mental health. ${ }^{8,23,24}$ While other studies have focused on efforts to reduce reliance on smartphones, the study reported herein was developed to understand how users respond to subtle actions 
designed to reduce the allure and attachment to smartphones. The theoretical basis for the design was based on the perceptual bias of salience that is often used in behavioral economics research. Salience describes the phenomenon that when one's attention is directed toward a particular portion of the environment, that portion will be elevated with regard to judgment and action. ${ }^{25}$ In other words, salience takes advantage of a bias that gives more weight and consideration to things brought to the forefront of our attention. Notification features (eg, ringing, vibrating, visual icons) of smartphones are prime examples of the salience principle to capture attention and entice use. Color also plays a role in overt attention ${ }^{26}$ and the presence of a smartphone near one's sleeping area increases the possibility of turning attention toward it during evening and early morning hours. Salience of one's smartphone and apps can theoretically be decreased by moving the device out of proximity of one's sleeping area, changing color settings to grayscale, removing social media apps from home screen, and turning off notifications. Based on the aforementioned research regarding salience, recommendations from the Center for Humane Technology, ${ }^{27}$ and ease of implementation, these four tasks were included in the wellness intervention described herein. Our study sought to elicit participant reactions, attitudes, and perceived effects that this behavioral economics intervention designed on the principle of salience had on their daily lives and personal well-being. We wanted to know what the participants actually felt, perceived, and thought while they were going through the study in order to better understand how this type of intervention might be used to reduce users' subconscious attraction to smartphones.

\section{METHODS}

During the spring semesters of 2019 and 2020, students enrolled in a required third year management course in the professional doctor of pharmacy program at the University of Kentucky were invited to participate in a series of optional challenges designed to enhance individual well-being. ${ }^{28}$ The first challenge, which is the focus of this study, lasted approximately 3 weeks at the beginning of the semester and involved actions to reduce psychological attachment to smartphones. Specifically, this challenge required participants to a) change default color setting for smartphone screen to grayscale, b) disable notifications for social media apps, c) remove social media icons from the home screen and d) not sleep with or near their smartphone. Additionally, completion of an anonymous reflective questionnaire administered via Canvas learning management system (Instructure, Inc, Salt Lake City, UT) was required to receive one extra credit point (equivalent to $0.2 \%$ of overall grade). The focus of the study reported here was to investigate participant reactions to the challenge's effects on their lives as indicated by the following questionnaire item. "Describe any positive or negative changes to your life and/or well-being that you noticed as a direct result of participating in this challenge? (open response)." The qualitative study was designed to better understand the depth of experiences of participants during the challenge, as opposed to determining precise effects of the different elements.

We used a thematic analysis protocol as recommended by Braun and Clarke ${ }^{29}$ to analyze questionnaire responses. Rather than pre-identify themes to search for, this design allows for themes to emerge from the data, which may reduce investigator bias. This method used an iterative process that involved familiarization with the data, identifying concepts expressed in the data, forming an initial set of themes, reviewing and refining themes, and coding the data with themes. Specifically, each of the three investigators individually read all participant responses while making personal notes of the concepts, ideas, and experiences expressed. As a group, the investigators discussed each of those concepts and categorized them into an initial set of themes that reflected a general sense of what participants articulated in their responses. For calibration reasons, investigators then individually used those themes to code a random $10 \%$ of responses before meeting again as a group to revise, refine, clarify, and finalize the set of themes to be used in the analysis. Each theme was given a name and description that explained attributes of the theme. Investigators then used this new set of themes to individually code all responses before meeting as a group to reconcile any differences. During the coding process, each participant response was assigned one or more themes as applicable. Every individually assigned theme that was not unanimous was discussed until consensus was reached. During the reconciliation process, one theme was split into two in order to more accurately reflect the meaning of participant responses. This study received exempt approval from the [University name redacted] Institutional Review Board.

\section{RESULTS}

One hundred eighty one of 274 students (66\%) participated in the smartphone well-being challenge. Eight responses were removed from the analysis because participants indicated that s/he did not complete all requirements of the challenge. Demographic information was not collected, but the average age of the student population in those classes was 25 and $67 \%$ were female. Twenty unique themes emerged from the analysis of the 173 responses. A total of 588 themes were assigned because responses often encompassed elements of more than 1 of the 20 themes. Table 1 contains all 
themes along with their descriptions, frequencies, and illustrative responses. Five themes were expressed in $25 \%$ or more of responses and 8 were expressed in $10-24 \%$ of responses.

\section{DISCUSSION}

This study sought to determine effects that turning phone to grayscale mode, removing social media icons from home screen, turning off notifications, and not sleeping with phone nearby had on students' daily lives and personal wellbeing. Results provide new information regarding the design of interventions to reduce smartphone and/or social media use in order to improve well-being. Approaches that direct participants to voluntarily restrict use have the potential for withdrawal symptoms, while our methods attempted to diminish the appeal of the device and associated media. ${ }^{30}$ The qualitative analysis of participant descriptions of any positive/negative changes to their lives resulting from the challenge revealed a variety of detailed perceptions of the effects. Overall, student reactions to their experience with this intervention were positive. Themes revealed that participants experienced several benefits with regard to time, productivity, sleep, interpersonal communications, and general well-being. The themes (Table 1) themselves are relatively straightforward and easy to comprehend, therefore the remainder of this discussion will focus on what these results mean with respect to existing and future research.

\section{Decreased Use of Smartphones and Social Media}

The large percentage of students who reported a decrease in smartphone use was expected but is also an important finding. As opposed to relying on willpower and self-discipline to reduce non-productive use of phones, the components of the challenge were designed to alleviate the primary attraction and attention to their smartphone. The themes expressed by students appeared to support that this was an effective way to reduce smartphone usage for a large percentage of participants. The decreased use of social media was likely a major reason why overall smartphone use was down.

Turning social media notifications off was alluded to as a factor contributing to decreased smartphone use. "I noticed that when I turned off social media notifications, I was less frequently checking the apps. " Without notifications, salience is reduced, and less attention is directed toward the apps. Previous research has shown that notifications provide external cues, which lead to increased phone/app checking. ${ }^{31}$ While our study did not attempt to analyze the direct effects of notifications on smartphone and social media use, the results align with research conducted by Du et al. ${ }^{32}$ which showed that notifications predict failure to self-control social media use. Social media notifications can be particularly disruptive to users who lack an awareness of the effect or who have poor self-regulatory behaviors. ${ }^{33}$ The failure to selfregulate increases the risk of addictive smartphone behavior. ${ }^{34,35}$ The measures used to reduce salience may have been more powerful toward those with lower self-regulation capabilities because it removed the frequency of which selfregulation was required.

A few participants $(11 \%)$ noted that the elements of the challenge made them more aware of their smartphone usage patterns that led them to regulate use. "I became conscious of how much time I spent on it every day and throughout the challenge, I was able to use the phone less and less." This was a somewhat surprising finding as the design of the challenge did not seek to increase mindfulness. However, mindfulness has been shown to have a positive effect on mental health factors resulting from problematic smartphone use and our results potentially provide insight into those findings. ${ }^{36}$ A considerable number of participants explicitly noted specific actions to regulate their smartphone usage. Twenty percent of participants expressed that they would continue some or all aspects of the challenge and $9 \%$ stated they took additional measures to reduce usage, such as setting timers, deleting apps, etc. While this study did not try to elicit why students used their phones and social media less, some of the other major themes discussed below provide insight.

\section{Grayscale's Effect on Desire and Use of Smartphone}

A second major finding of this study was the effects of a default grayscale setting. Participants alluded to grayscale as a factor that reduced the attraction of casually browsing social media, indicating that session times on social media may have decreased. "I noticed that the grey scale on my phone really did make me want to put it down faster. I noticed spending less time on social media because I was frustrated about not being able to see the pictures in color." Specifically, the visually oriented platform of Instagram was mentioned numerous times as being less interesting. Although the popular press ${ }^{37}$ is replete with articles extolling benefits of grayscale mode on reducing social media use, research in this area has been limited. The one published study to specifically examine grayscale mode showed that undergraduate students who changed their phones to grayscale significantly reduced their screen time by an average of 37.9 minutes each day. ${ }^{38}$ The results of our study provide further evidence that grayscale alters the user experience and reduces the appeal of social media use on smartphones. 
In this analysis, a distinction was made between grayscale mode reducing the allure to a smartphone and the frustrating effects that grayscale mode had on usage. Several participants cited that grayscale was frustrating because it hampered their ability to use the smartphone productively for activities relying on color (eg, calendars, viewing charts/graphs, etc.) "However, it was a little frustrating when doing certain tasks related to looking at pictures or house hunting." Still others commented on the noticeable differences that grayscale seemed to have on their physical health. Some pointed to headaches and eyestrain, while others cited a positive effect on their vision. These different responses to grayscale indicate that its effect on reducing smartphone usage is mixed. For many, it is potentially an effective way to remove the appeal of smartphones. However, for others the negative effects on usability and physical health reduce the likelihood that this would be an effective long-term solution to curbing individual smartphone usage.

\section{Positive Effects on Overall Observed Well-being}

One of the underlying reasons for conducting this study was to learn if simple measures to reduce smartphone use might affect perceptions of personal well-being. Results indicate that there were benefits to well-being for most participants. In addition to several of the themes that either relate to or are factors of well-being, $15 \%$ of the responses made direct reference to improvements in overall stress, anxiety, depression, etc. "Keeping the notifications off in particular decreased my anxiety level." The exact cause(s) of the improved well-being is unknown as it could potentially be any combination of factors pertaining to distractions, sleep, interpersonal communications, social comparisons, etc. Previous research has yielded mixed results with regard to smartphone/social media use and mental health factors. While numerous studies have reported a negative relationship between digital device/media use and well-being, the overall effect sizes are small. ${ }^{7,839}$ Additional studies are necessary to determine the nuances between smartphone use and mental health.

Related to well-being, another notable theme expressed by participants was that the smartphone challenge positively affected their face-to-face interactions with others. Pointing to the fact that they were less distracted by their phone, participants observed marked improvement in their in-person relations and communications with those around them. One participant illustrated this point in their response "The biggest positive change is that my wife and I found that we spent much less time on our phones and became more connected/enjoyed our time together more!" This theme adds credence to previous research showing that smartphone distractions can hamper enjoyment, personal connections, and satisfaction with others. ${ }^{19,40-43}$

Another well-being related finding of this study was the positive effects on sleep. "I found keeping my phone away from my bed helped me fall asleep before midnight, rather than staying up until 1-2 a.m. looking at random things that caught my attention from social media." Twenty percent of all reflective responses referred to improved sleep quality and/or quantity, which is not surprising given that numerous research studies have found negative correlations between smartphone usage and sleep. ${ }^{44-46}$ Furthermore sleep quality is predictive of positive affect, which may have contributed to our findings regarding perceived improvements in overall well-being. ${ }^{47}$

\section{Neutral and/or Negative Effects of the Activities}

While themes derived from participant reactions revealed overall positive effects, a few responses expressed neutral or negative effects. Two of the lesser-occurring themes revealed no noticeable change (3\%) or simply that participants started using other digital devices (eg, laptops, tablets, etc.) more to compensate (6\%).

In addition to eyestrain and frustrating aspects of grayscale on productivity-related tasks, more than $10 \%$ of responses referred to a fear of missing communications or alarms as a result of turning notifications off and/or not sleeping near their phone. "A negative change that I noticed was I was sometimes scared I was going to "miss something'." This result is not surprising as fear of missing out (FOMO) has been shown to be an antecedent to problematic smartphone use. ${ }^{48}$ The themes indicative of negative responses provides insight into how smartphones are interwoven into people's daily activities, including ways that can be simultaneously positive and negative. Unlike other "vices" that have no redeemable qualities, smartphones play an important role in the functioning of modern society. ${ }^{49}$ Because of all the positive uses for smartphones, users may concentrate on beneficial uses while ignoring the potential harms. ${ }^{19,50}$ This can make it more challenging to address problems associated with smartphones and any intervention that seeks to limit or alter smartphone use should also consider potential unintended effects.

\section{Implications}

Results of this study can be used when considering measures to reduce smartphone usage in order to improve facets of mental health. Because of the aforementioned inability of many to self-regulate their own usage, recommendations based upon elements of the challenge described herein may be more effective because salience of (and 
thus attention to) the smartphone is reduced. It may be more effective to teach smartphone users how to psychologically circumvent the mechanisms that draw attention to the phones than to simply encourage them to use smartphones less.

\section{Limitations}

The study has a few limitations. First, there is potential for a sampling bias as the study was conducted on an optional opt-in basis. Certain characteristics that affect one's psychological connection to smartphones or general wellbeing practices could be under- or over-represented in this sample. Second, the actions required by study participants only lasted a few weeks. A longer time period may result in differing, dissipated, or enhanced perceptions. Third, the study only analyzed participant responses to an open-ended question, therefore a participant's experience would only be encompassed in a theme if it was directly expressed in the response. Different methods such as focus group or individual interviews, could have prompted the participant to reveal more thoughts and perceptions than what they included in their initial response. Finally, due to the self-reporting nature of the study, participant responses could have been influenced by a social-desirability bias.

\section{Future research}

The results of this study lay the groundwork for future research that can aid the understanding of links between problematic smartphone use and well-being. First, using the emergent themes of this study, a quantitative approach is needed to document the extent that the outcomes (versus perceived outcomes) occur across an entire sample. Second, individual elements of the challenge (eg, grayscale mode, turning off notifications, etc.) should be examined independently in controlled experiments to quantify the effect on smartphone and social media usage and on different factors of well-being (ie, stress, anxiety, depression).

\section{CONCLUSION}

Pharmacy students who turned smartphone settings to grayscale, turned off notifications, removed social media icons from home screen, and slept away from their phones reported primarily positive outcomes. In many cases, less time on smartphones meant better sleep, more productivity, more time for social/leisure activities, improved face-to-face interactions, and/or improvement in well-being. Results of this study provide initial evidence that simple changes to smartphone settings and placements at night may produce positive outcomes for those who want to reduce time spent on smartphones.

\section{REFERENCES}

1. American Association of Colleges of Pharmacy. AACP Statement on Commitment to Clinician Well-being and Resilience. https://www.aacp.org/article/commitment-clinician-well-being-and-resilience. Published 2019. Accessed July 5, 2021.

2. Hagemeier NE, Carlson TS, Roberts CL, Thomas M. A longitudinal analysis of first professional year pharmacy student well-being. Am J Pharm Educ. 2020;84(7):Article 7735.

3. Brazeau GA, Frenzel JE, Prescott WA. Facilitating wellbeing in a turbulent time. Am J Pharm Educ. 2020;84(6):Article 8154.

4. Cain J. It's time to confront student mental health issues associated with smartphones and social media. Am J Pharm Educ. 2018;82(7):Article 6862.

5. $\quad$ Twenge JM. Have smartphones destroyed a generation? The Atlantic. https://www.theatlantic.com/magazine/archive/2017/09/has-the-smartphone-destroyed-a-generation/534198/. Published 2017. Accessed November 11, 2020.

6. Billieux J. Problematic use of the mobile phone: A literature review and a pathways model. Curr Psychiatry Rev. 2012;8(4):299-307.

7. Elhai JD, Dvorak RD, Levine JC, Hall BJ. Problematic smartphone use: A conceptual overview and systematic review of relations with anxiety and depression psychopathology. J Affect Disord. 2017;207:251-259.

8. Vahedi Z, Saiphoo A. The association between smartphone use, stress, and anxiety: A meta- analytic review. Stress Health. 2018;34(3):347-358.

9. Volkmer SA, Lermer E. Unhappy and addicted to your phone?- Higher mobile phone use is associated with lower well-being. Comp Human Behav. 2019;93:210-218.

10. Cain J, Kelley JL, Malcom D. A scoping review of the association between smartphone use and mental health among college students. Int J Depress Anxiety. 2020;3(2). 
11. Mac Cárthaigh S, Griffin C, Perry J. The relationship between sleep and problematic smartphone use among adolescents: A systematic review. Develop Rev. 2020;55:100897.

12. Demirci K, Akgönül M, Akpinar A. Relationship of smartphone use severity with sleep quality, depression, and anxiety in university students. J Behav Addict. 2015;4(2):85-92.

13. Elhai JD, Hall BJ, Levine JC, Dvorak RD. Types of smartphone usage and relations with problematic smartphone behaviors: The role of content consumption vs. social smartphone use. Cyberpsychol. 2017;11(2):Article 3.

14. Kardefelt-Winther D. A conceptual and methodological critique of internet addiction research: Towards a model of compensatory internet use. Comp Human Behav. 2014;31:351-354.

15. Firth J, Torous J, Nicholas J, et al. The efficacy of smartphone- based mental health interventions for depressive symptoms: a meta- analysis of randomized controlled trials. World Psychiatry. 2017;16(3):287-298.

16. Wiederhold BK. Using social media to our advantage: Alleviating anxiety during a pandemic. Cyberpsychol Behav Soc Netw. 2020;23(4):197-198.

17. McKenzie L. Stop me before I text again. Inside Higher Ed. https://www.insidehighered.com/news/2018/03/06/stanford-students-challenge-apple-iphone-addiction. Accessed July 6, 2021.

18. Jiang J. How teens and parents navigate screen time and device distractions. Pew Research Center for Internet and Technology. 2018.

19. Harkin LJ, Kuss D. "My smartphone is an extension of myself": A holistic qualitative exploration of the impact of using a smartphone. Psychol Pop Media. 2020;10(1):28-38.

20. Harris T. How technology Hijacks People's minds-From a magician and google's design ethicist. Medium Magazine. 2016.

21. Lewis P. 'Our minds can be hijacked': the tech insiders who fear a smartphone dystopia. The Guardian. 2017;6(10).

22. van Velthoven MH, Powell J, Powell G. Problematic smartphone use: Digital approaches to an emerging public health problem. In: SAGE Publications Sage UK: London, England; 2018.

23. Elhai JD, Levine JC, Hall BJ. Problematic smartphone use and mental health problems: current state of research and future directions. J Psychiatry Neurol Sci. 2019;32(1):1.

24. Kornberg J. Left to our own devices: Smartphone use, mental health, and academic psychiatry. Acad Psychiatry. 2020;44(4):483-486.

25. Taylor SE, Thompson SC. Stalking the elusive" vividness" effect. Psychol Rev. 1982;89(2):155.

26. Frey H-P, Honey C, König P. What's color got to do with it? The influence of color on visual attention in different categories. J Vis. 2008;8(14):1-17.

27. Center for Humane Technology. Take Control. https://www.humanetech.com/take-control. Accessed July 7, 2021.

28. Cain J. Effectiveness of issuing well-being challenges to nudge pharmacy students to adopt well-being protective behaviors. Am J Pharm Educ. 2020;84(8):Article 7875.

29. Braun V, Clarke V. Using thematic analysis in psychology. Qual ResPsychol. 2006;3(2):77-101.

30. Stieger S, Lewetz D. A week without using social media: Results from an ecological momentary intervention study using smartphones. Cyberpsychol Behav Soc Netw. 2018;21(10):618-624.

31. Kim M. The effects of external cues on media habit and use: Push notification alerts and mobile application usage habits. Michigan State University; 2014.

32. Du J, Kerkhof P, van Koningsbruggen GM. Predictors of social media self-control failure: Immediate gratifications, habitual checking, ubiquity, and notifications. Cyberpsychol Behav Soc Netw. 2019;22(7):477-485.

33. Wu J-Y. University students' Motivated Attention and use of regulation strategies on social media. Comp Educ. 2015;89:75-90.

34. Gökçearslan Ş, Mumcu FK, Haşlaman T, Çevik YD. Modelling smartphone addiction: The role of smartphone usage, self-regulation, general self-efficacy and cyberloafing in university students. Comp Human Behav. 2016;63:639-649.

35. Van Deursen AJ, Bolle CL, Hegner SM, Kommers PA. Modeling habitual and addictive smartphone behavior: The role of smartphone usage types, emotional intelligence, social stress, self-regulation, age, and gender. Comp Human Behav. 2015;45:411-420.

36. Apaolaza V, Hartmann P, D'Souza C, Gilsanz A. Mindfulness, compulsive mobile social media use, and derived stress: The mediating roles of self-esteem and social anxiety. Cyberpsychol Behav Soc Netw. 2019;22(6):388-396.

37. Bowles N. Is the answer to phone addiction a worse phone. The New York Times. Jan 11, 2018, 2018. 
38. Holte AJ, Ferraro FR. True colors: Grayscale setting reduces screen time in college students. Soc Sci J. 2020:117.

39. Orben A, Przybylski AK. The association between adolescent well-being and digital technology use. Nat Hum Behavr. 2019;3(2):173-182.

40. Dwyer RJ, Kushlev K, Dunn EW. Smartphone use undermines enjoyment of face-to-face social interactions. $J$ Exp Soc Psychol. 2018;78:233-239.

41. Przybylski AK, Weinstein N. Can you connect with me now? How the presence of mobile communication technology influences face-to-face conversation quality. J Soc Pers Relat. 2013;30(3):237-246.

42. Rotondi V, Stanca L, Tomasuolo M. Connecting alone: Smartphone use, quality of social interactions and wellbeing. J Econ Psychol. 2017;63:17-26.

43. Vogels EA, Anderson M. Dating and relationships in the digital age. https://www.pewresearch.org/internet/wpcontent/uploads/sites/9/2020/05/PI_2020.05.08_dating-digital-age_REPORT.pdf. Published 2020. Accessed March 1, 2021.

44. Boonluksiri P. Effect of smartphone overuse on sleep problems in medical students. Asia Pacific Schol: Med Health Profess Educ. 2018;3(2):25-28.

45. Lemola S, Perkinson-Gloor N, Brand S, Dewald-Kaufmann JF, Grob A. Adolescents' electronic media use at night, sleep disturbance, and depressive symptoms in the smartphone age. Journal Youth Adolesc. 2015;44(2):405-418.

46. Wang P-Y, Chen K-L, Yang S-Y, Lin P-H. Relationship of sleep quality, smartphone dependence, and healthrelated behaviors in female junior college students. PloS one. 2019;14(4):e0214769.

47. Bouwmans ME, Bos EH, Hoenders HR, Oldehinkel AJ, de Jonge P. Sleep quality predicts positive and negative affect but not vice versa. An electronic diary study in depressed and healthy individuals. J Affect Disord. 2017;207:260-267.

48. Yang Z, Asbury K, Griffiths MD. “A cancer in the minds of youth?” A qualitative study of problematic smartphone use among undergraduate students Int J Ment Health Addict. 2019:1-13.

49. Nath A, Mukherjee S. Impact of Mobile Phone/Smartphone: A pilot study on positive and negative effects. Int $J$ Adv Res Comp Sci Manage Studies. 2015;3(5):294-302.

50. AlBarashdi HS, Bouazza A, Jabur NH, Al-Zubaidi AS. Smartphone addiction reasons and solutions from the perspective of sultan qaboos university undergraduates: A qualitative study. Int J Psychol Behav Analy. 2016;2(113). 


\begin{tabular}{ll}
\hline \multicolumn{1}{c}{ Theme } & \multicolumn{1}{c}{ Description } \\
\hline Smartphone use decreased & $\begin{array}{l}\text { Participant's overall time spent on smartphone } \\
\text { decreased } \\
\text { Grayscale made the phone boring and reduced the } \\
\text { desire to look at it }\end{array}$ \\
Social media use decreased & Participant's overall time on social media decreased \\
Grayscale was frustrating & $\begin{array}{l}\text { Grayscale created frustration and/or discontent when } \\
\text { performing activities on the phone in which color } \\
\text { played an important role }\end{array}$ \\
Productivity increased & $\begin{array}{l}\text { Participant able to focus on school and/or work } \\
\text { activities due to checking phone less frequently }\end{array}$ \\
Attachment to phone decreased & $\begin{array}{l}\text { Participant felt less attached to their phone } \\
\text { Participant plans to continue certain aspects of the } \\
\text { challenge after it is over }\end{array}$ \\
Sleep improved & $\begin{array}{l}\text { Participant fell asleep easier, slept more, and/or sleep } \\
\text { quality improved }\end{array}$ \\
Distractions from phone decreased & Phone became less distracting to participant
\end{tabular}

"Example response

$41 \%$, then $11 \%$, and then $14 \%$ "

"Using gray-scale mode made me feel like my life was a melodramatic series except much less interesting"

"I immediately noticed a decrease in the amount of time I spent on my phone using social media."

"However, it was a little frustrating when doing certain tasks related to looking at pictures or house hunting."

"I noticed that I was more productive when studying and working on things because I felt less tempted to reach for my phone every 15 minutes."

"I felt less tethered to my phone"

"I'm going to continue leaving my notifications off and not sleeping by my phone."

"(M)y sleep was better! On average I think I gained an hour of sleep every day"

"I found that without these constant notifications, I was less tempted to check these apps regularly. For example, I would go much longer without going on Facebook."

"There was a lot less of the comparison game and negative feelings towards myself. I can't explain how much of a positive impact removing myself from these things has had on me mentally!"

"A negative change that I noticed was I was sometimes scared I was going to "miss something." The grayscale mode also impacted this the most. Whenever I was on my phone things did not stand out near as much as they did before. Therefore, I was concerned I was going to miss an important email, social media post, or any other important news, etc. As far as I know, I never missed anything, but there was that tiny worry in the back of my mind from time to time."

"I found myself interacting more with the people around versus holding my phone and attempting to look at my phone and hold a conversation."

"This challenge made me realize just how much I have let my phone take up so much of my time that I could be spending doing so many better things. I

$\mathbf{N}(\%)$

$90(52 \%)$

$58(34 \%)$

Participant paid more attention to those in their improved

Mindfulness of phone and social media use
Participant became aware of time spent on phone and/or social media 


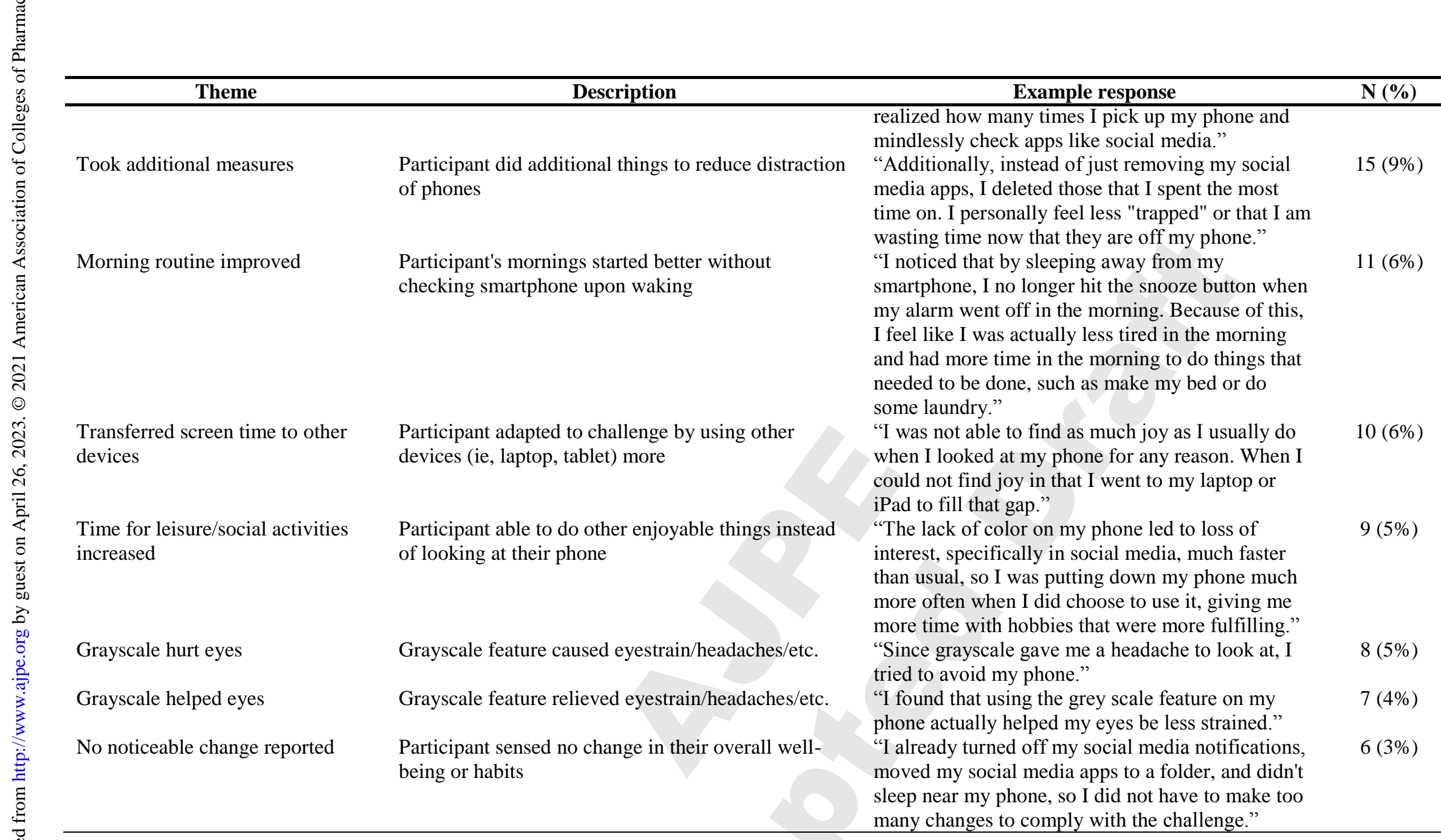

